\title{
Nanocosmeceuticals: Latest Trend in Cosmetics
}

\author{
Aishwarya Kashyap, Prakhar Agarwal, Monika Soni * \\ Rapture Biotechnology, Bhopal, India
}

Address for Correspondance: Monika Soni, aishkashgr8@gmail.com ; aprakhar50@gmail.com ; info.rapturebiobhopal@gmail.com

\begin{abstract}
Keywords
Cosmeceuticals;

Nanotechnology;

Pharmaceuticals;

Nanocarriers;

Nanocosmeceutical

S.
\end{abstract}

\begin{abstract}
Cosmeceuticals" is a word that defines a product which has its biological action like a pharmaceutical drug and it also regulates the appearance as a cosmetic. They are present to enhance the beauty of the skin through ingredients that provide additional health-related benefit. Cosmeceutical industry is growing day by day as it is highly diversified, since it serves as a link between personal care products and pharmaceuticals. Use of nanotechnology in the field of cosmeceutical industry is an evolving area of research and development. Nano cosmeceuticals overcomes many disadvantages associated with cosmeceuticals like they improve the stability of cosmetic ingredients by encapsulating them within the nanoparticles, UV protection of skin, targeting of active ingredient to the desired site and controlled release for prolonged effect on the skin. Nanoproducts should be manufactured in a manner that improves the values of cosmeceutical product. Here is a brief overview of the various novel nano-carriers for cosmeceuticals like liposomes, nanoemulsions, solid lipid nanoparticles(SLNs), dendrimers, inorganic nanoparticles, and many others. The review also talks about the possible hazards of nanoparticles on human health but they can be neglected due to less significant toxicity. (C) 2016 iGlobal Research and Publishing Foundation. All rights reserved.
\end{abstract}

Conference Proceedings: International Conference on Advances in Plant and Microbial Biotechnology (PMB2017); JIIT, Noida: February 02-04, 2017

Indo Global Journal of Pharmaceutical Sciences( ISSN 22491023 ; CODEN- IGJPAI; NLM ID: 101610675) indexed and abstracted in EMBASE(Elsevier), SCIRUS(Elsevier),CABI, CAB Abstracts, Chemical Abstract Services(CAS), American Chemical Society(ACS), Index Copernicus, EBSCO, DOAJ, Google Scholar and many more. For further details, visit http://iglobaljournal.com 